\title{
Late-onset epilepsy associated with untreated obstructive sleep apnea: A case report and literature review
}

\author{
Cheng J $Y^{*}$ \\ Assistant Professor, Department of Neurology, NYU School of Medicine, New York
}

\begin{abstract}
Approximately one-quarter of people with epilepsy will experience their first seizure in later adulthood, and up to almost $40 \%$ of these individuals will have no clear seizure etiology despite extensive investigation. Obstructive sleep apnea (OSA), which becomes increasingly prevalent with age, may contribute to seizure facilitation in susceptible individuals. OSA treatment has been demonstrated to decrease interictal activity and improve seizure control, and as a modifiable risk factor, should be evaluated in patients with late-onset seizures. The following case describes a patient who presented with overt symptoms of late-onset epilepsy after being diagnosed with OSA, with further seizure exacerbation during noncompliance with continuous positive airway pressure therapy.
\end{abstract}

\section{Introduction}

The prevalence of epilepsy ranges from 0.5 to $1 \%$ [1]. Approximately $25 \%$ of these individuals will experience their first seizure in adulthood, with a peak in incidence for those aged 65 years and older [2,3]. The etiology of $20-38 \%$ of adult onset epilepsy remains unclear despite extensive investigation, but precipitating factors, including untreated obstructive sleep apnea (OSA), have been associated with seizure facilitation in susceptible populations [4]. Posited mechanisms of seizure facilitation in patients with untreated OSA include sleep fragmentation, cerebral hypoxemia, and cardiac dysfunction [4]. Treatment of OSA with continuous positive airway pressure (CPAP) has been demonstrated to improve seizure control $[5,6]$, as well as to decrease interictal activity [7], supporting the role of untreated OSA in seizure facilitation. This suggests that, as a modifiable risk factor, OSA should be evaluated in patients with late-onset seizures. The following case describes a patient who presented with late-onset epilepsy after being diagnosed with OSA, with further seizure exacerbation during noncompliance with CPAP, and highlights the importance of recognizing and treating OSA in late-onset epilepsy.

\section{Case Report}

A 62-year-old right-handed man presented to a university sleep center for evaluation of loud snoring, daytime sleepiness, and multiple arousals at night, which had become worse over several years. His past medical history was significant for obesity, hypertension, hyperlipidemia, and post-traumatic stress disorder (PTSD), which was being successfully managed by weekly group therapy sessions. There was no history of tobacco, alcohol or illicit drug abuse. Medications included aspirin, metoprolol, fenofibrate and rosuvastatin. Epilepsy risk factors were negative for prematurity, developmental delay, febrile convulsions, and family history of epilepsy, and significant for an episode of loss of consciousness lasting several minutes after a motor vehicle collision in 1992, which did not require hospitalization. On general physical exam, the patient was obese (body mass index $=31$ ), with a normal neck circumference and modified Mallampati index $=2$. Cardiac, respiratory and neurological exams were nomal.
Attended in-lab polysomnography (PSG) was performed, using a three channel EEG montage (F4-M1, C4-M1, and O2-M1) and electrooculogram. PSG demonstrated loud snoring and an apnea-hypopnea index (AHI) of 17, indicative of moderate obstructive sleep apnea. Sleep efficiency and percentage of sleep in REM and NREM (nonREM) stages was normal. Wake after sleep onset was 23.5 minutes. Less than 1 minute of recording time was spent below $90 \%$ oxygen saturation. The periodic limb movement index was not elevated, and no EKG abnormalities were present.

After a titration study, the patient was started on CPAP at a pressure of $6 \mathrm{~cm} \mathrm{H20,} \mathrm{with} \mathrm{resolution} \mathrm{of} \mathrm{snoring,} \mathrm{nighttime} \mathrm{arousals,} \mathrm{and}$ improved daytime sleepiness. He was compliant with CPAP therapy, stating that it was difficult to fall asleep without using the machine, and averaged 6-8 hours of usage per night, 7 days per week.

Four months later, the patient's wife witnessed an episode of unresponsiveness from sleep. This occurred after technical problems prevented the patient from using his CPAP machine for the prior two evenings. The wife was initially awoken by gurgling sounds from the patient during the first half of the night and observed foaming at the mouth. The patient's eyes were open, but he was not responsive to questions. During this event, he sat up and briefly spoke meaningless words, with deviation of the eyes and head towards the left. Bowel and bladder incontinence were not present. Emergency medical services were called, and the patient regained awareness during the ambulance ride to the hospital approximately 20 minutes after the beginning of the observed event. He was amnestic of the episode, and it was not associated with vivid dreaming. Subsequent evaluation of this event at the outside hospital consisted of an unremarkable MRI brain without contrast (Figures 1 and 2), normal 24-hour Holter monitoring,

Correspondence to: Cheng JY, MD, Assistant Professor, NYU Comprehensive Epilepsy Center, Department of Neurology, NYU School of Medicine, 223 E. 34th Street, New York, NY 10016, Email: jocelyn.cheng@nyumc.org

Key words: seizures, epilepsy, obstructive sleep apnea, late-onset epilepsy, adultonset epilepsy

Received: March 29, 2018; Accepted: April 11, 2018; Published: April 14, 2018 


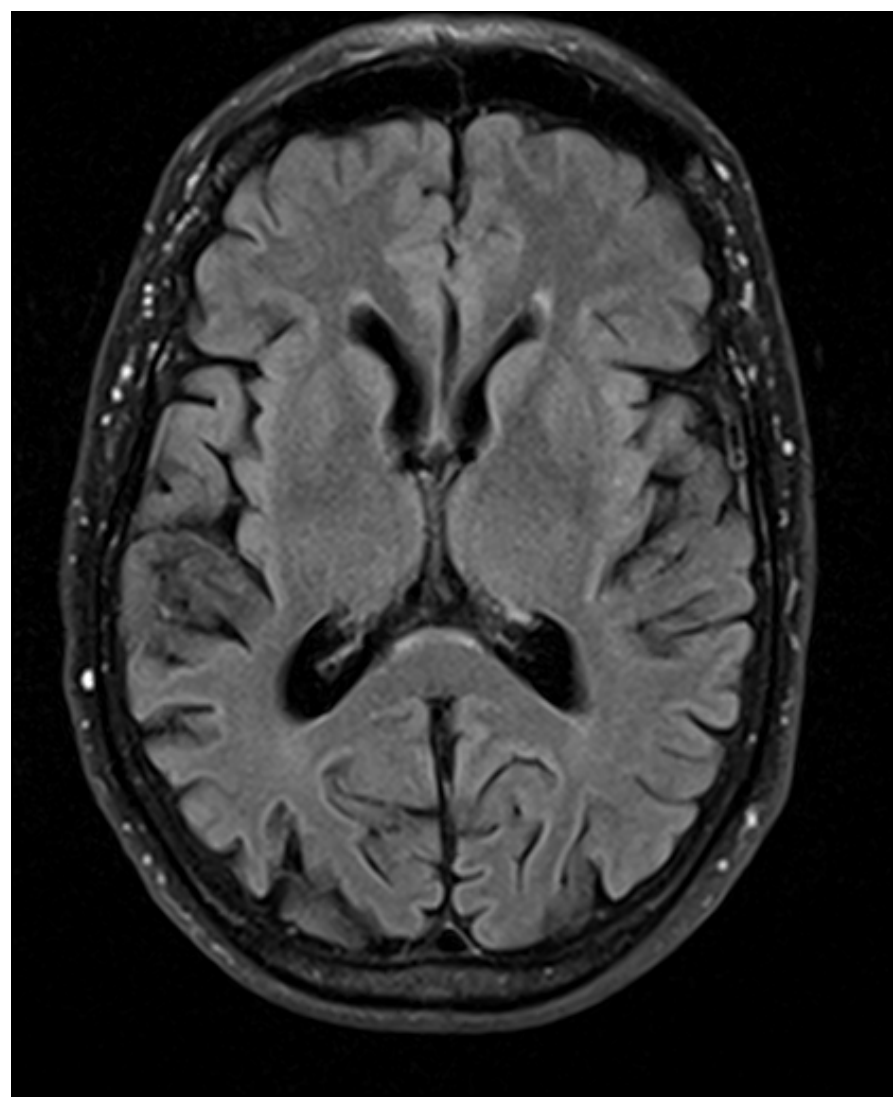

Figure 1. MRI brain without contrast: Axial FLAIR.

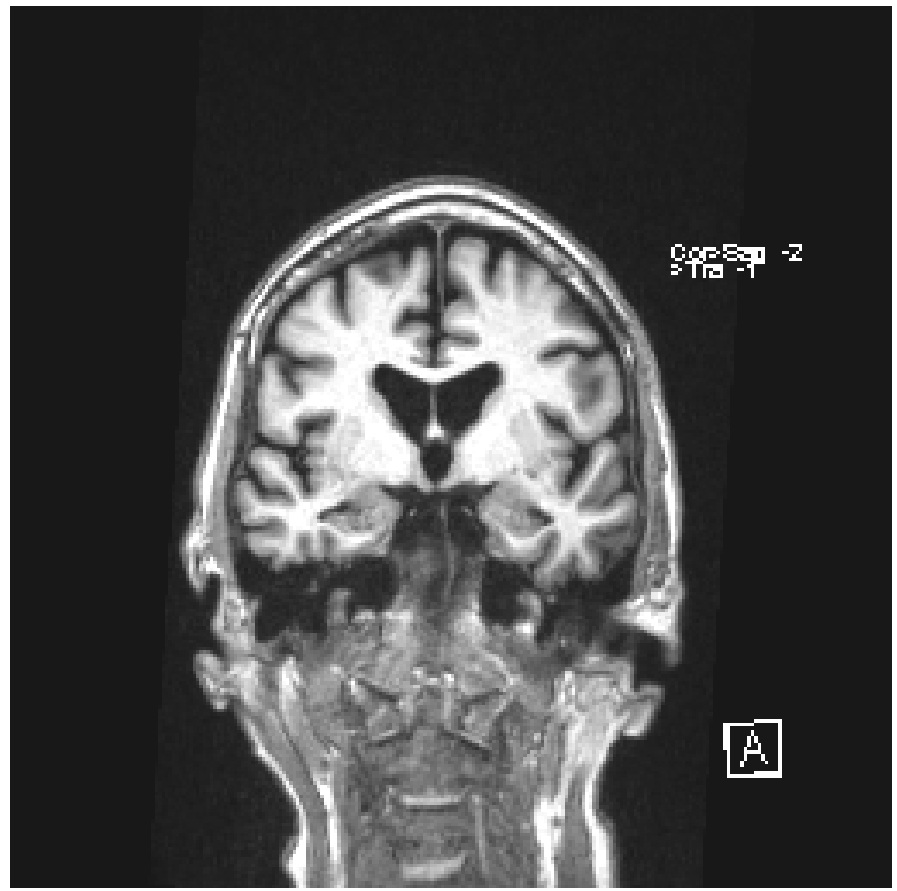

Figure 2. MRI brain without contrast: Coronal T1.

and normal routine EEG. As the patient's exam and work-up were unremarkable, and this was his first episode of impaired awareness, no intervention was started.
One year after his OSA diagnosis, the patient returned to the university sleep center for routine follow-up. His sleep remained refreshing, and CPAP use averaged 6-8 hours per night, every night. However, he reported that two episodes of unresponsiveness had occurred in the interim since he was last seen. Upon further interview, the patient stated that he had been experiencing transient episodes of anxiety/fear, sometimes associated with abdominal discomfort, for a few years. The sensation would last for a few seconds, and occurred approximately once per month, without clear precipitating factors. Similar to the first unresponsive episode which occurred four months after OSA was diagnosed, the second episode was associated with not using CPAP the previous evening, secondary to returning home at so late an hour that the patient fell asleep before using the machine. The patient's wife was again awoken by gurgling sounds during the first half of the night, and witnessed him foaming at the mouth, eyes open. He did not respond to questions. His eyes and head were again noted to be deviated to the left. There was no bowel or bladder incontinence. Emergency medical services were once again called, and the patient regained baseline awareness in the ambulance approximately 30 minutes after observed episode onset. There was no associated vivid dreaming, and he was amnestic of the event. Laboratory studies and a noncontrast head CT performed in the emergency room were normal.

A sleep-deprived, prolonged ( $>60$ minutes) EEG was ordered, which showed interictal sharp waves in the right frontotemporal region at F8 (Figure 3). He subsequently underwent inpatient video EEG monitoring, during which time a focal seizure of right frontotemporal onset with secondary generalization was captured, associated with the same clinical characteristics as described in his prior two nocturnal episodes of impaired awareness (Figures $4 \mathrm{a}-4 \mathrm{c}$ ). He was diagnosed with focal epilepsy and started on levetiracetam $500 \mathrm{mg}$ by mouth twice daily.

Thirteen months after initiation of antiseizure medication (ASM), the patient's focal aware seizures of epigastric discomfort and anxiety/ fear had decreased in frequency, from monthly to every 4-6 months. There were also no further episodes of impaired awareness, even when he missed using CPAP on rare occasions.

\section{Discussion}

Obstructive sleep apnea (OSA) has an estimated prevalence of $2 \%$ in women and $4 \%$ in men [8], with increasing prevalence in older individuals as they approach the age of 65 years [9]. OSA is present in $5-63 \%$ of people with epilepsy (PWE) [10], and of PWE, approximately $25 \%$ will experience their first seizure in adulthood. As epilepsy and OSA are common in the older population, their co-occurrence may be coincidental. However, the older brain carries an increased susceptibility to insult due to the burden of co-morbid disease with aging. While co-morbidities such as ischemic vascular disease and post-traumatic brain injury have been attributed to late-onset seizures, this disease burden alone does not account for the etiology of $20-38 \%$ of patients with adult onset epilepsy [2,3]. Several studies suggest that untreated OSA facilitates seizures in susceptible patient populations, particularly older individuals. Manni et al demonstrated that older age at seizure onset was the only epilepsy risk factor found to be significantly associated to OSA in a group of epilepsy patients [11]. A cross-sectional study by Chihore, et al. showed complementary results, in that patients with seizure onset at age $\geq 50$ years or with greater seizure frequency at or after 50 years of age had significantly higher AHIs [12]. This suggests that a subset of late-onset epilepsy patients is susceptible to seizure facilitation due to untreated OSA. 


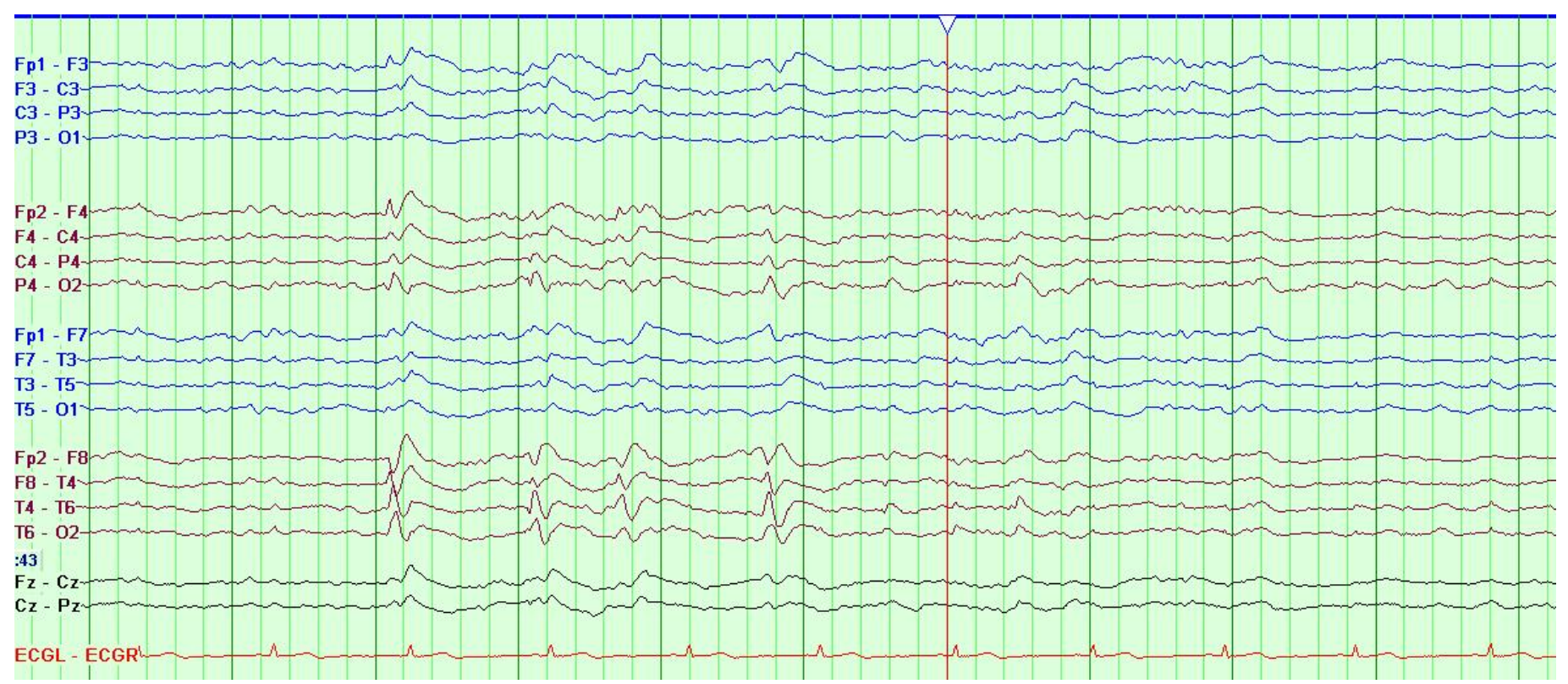

Figure 3. Sleep-deprived EEG demonstrating interictal sharp waves in the right frontotemporal region at F8.

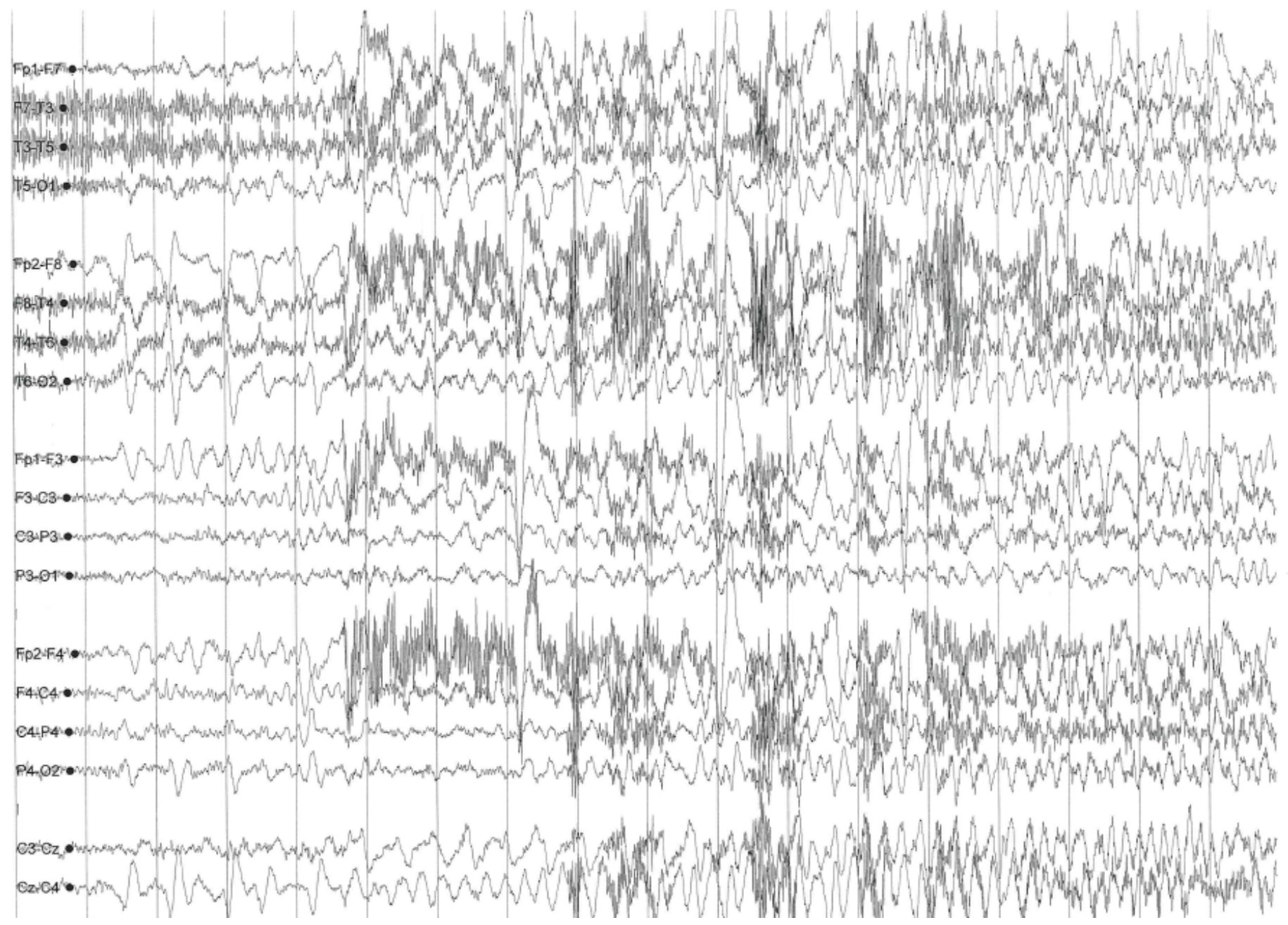

Figure 4a. Focal seizure with secondary generalization of right frontotemporal onset. 
A

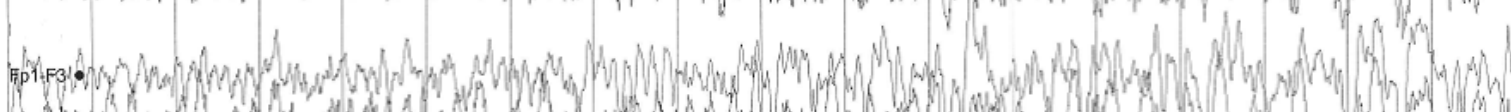

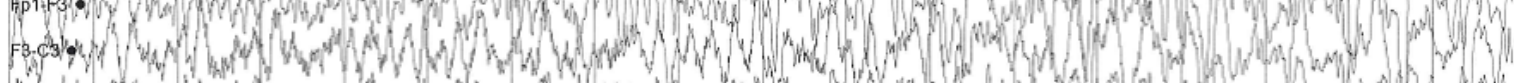

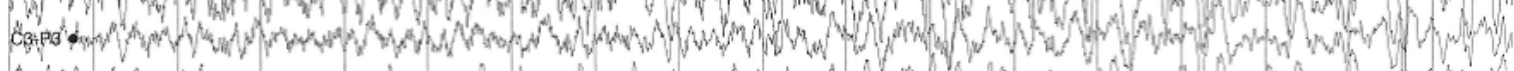

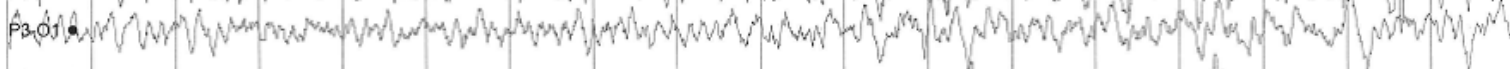

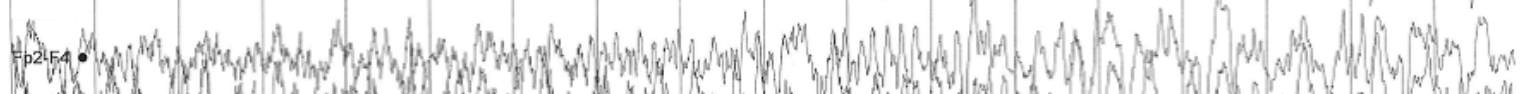

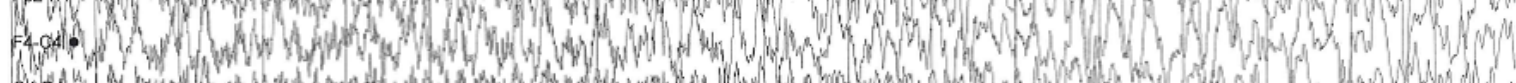

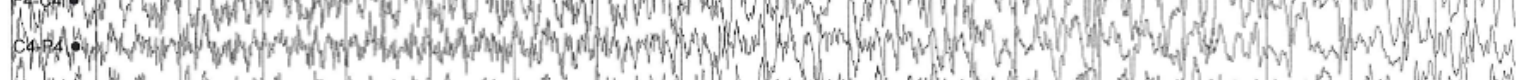
P4.0

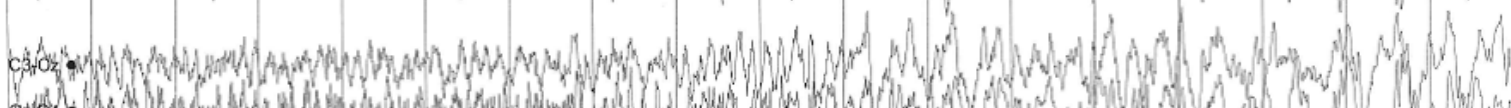

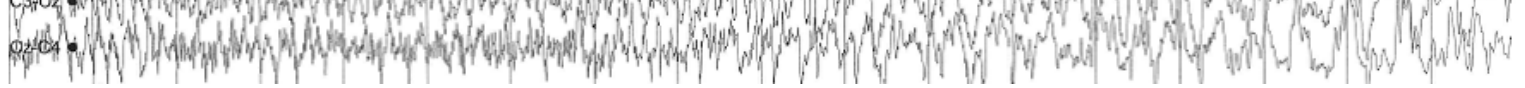

Figure 4 b. Focal seizure with secondary generalization of right frontotemporal onset.

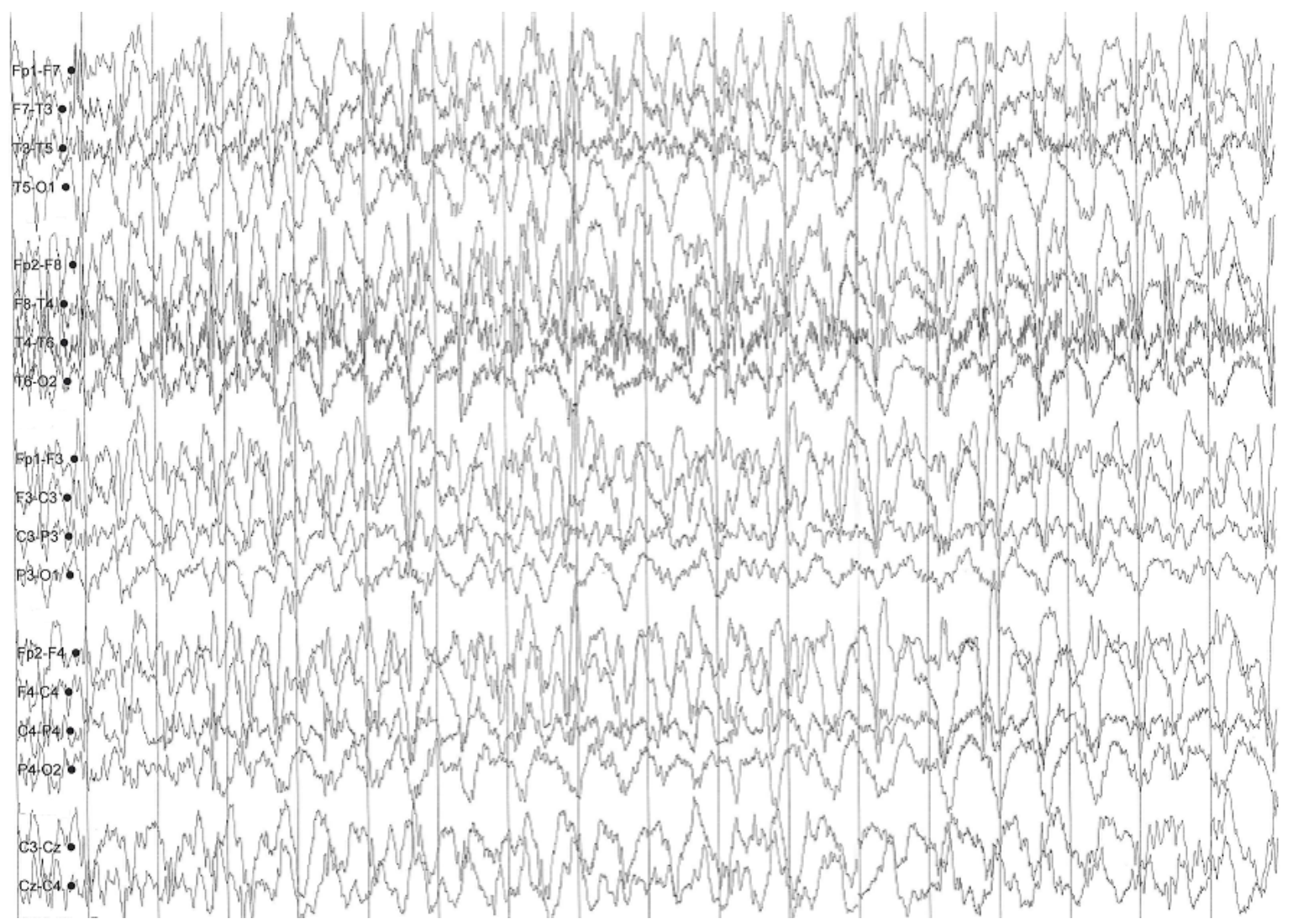

Figure 4c. Focal seizure with secondary generalization of right frontotemporal onset. 
The literature also supports the relationship between untreated OSA and seizures. In one series, the seizure frequency of seven patients with refractory epilepsy and sleep apnea was followed before and after apnea treatment (CPAP, tracheostomy or medical therapy). Seizure frequency and severity was reduced in 6 of 7 patients, even with intermittent CPAP use [4]. In a retrospective review by Hollinger's group, 29 patients with epilepsy and OSA were identified. Twenty-three of these patients were prescribed CPAP therapy, and good compliance ( $\geq 4$ hours of use per night) was obtained in 12 patients. Four out of 12 patients showed a clear reduction in seizure frequency, with one patient becoming seizure free with CPAP alone, i.e. without initiation of an ASM [10]. In another retrospective review, CPAP compliance was studied in 41 patients with OSA and epilepsy for a minimum of 6 months. The compliant group, comprised of 28 patients, demonstrated a significant decrease in monthly seizure frequency, while the 13 in the noncompliant group showed no change [13]. A pilot study by Malow et al assigned adult patients with refractory epilepsy and OSA to therapeutic or sham CPAP. Seizure frequency was reduced by $50 \%$ or greater in $28 \%$ of patients on therapeutic CPAP versus $15 \%$ in the sham group, although this was not statistically significant [6]. Vaughn's group described a series of 10 adult patients with recurrent seizures and OSA who were prescribed CPAP or positional therapy. With apnea treatment alone, 3 patients became seizure free, and 1 had a greater than $95 \%$ reduction in seizure frequency. Three patients required OSA treatment and ASM titration for improved seizure control, and three patients had a seizure reduction of less than $50 \%$, one of whom was unable to tolerate CPAP [5].

Electroencephalographic evidence of decreased interictal activity has also been demonstrated. Oliveira studied the interictal activity rate of epilepsy patients, six with OSA and two with COPD and snoring, before and after CPAP. All patients demonstrated a reduction in respiratory disturbance index and spiking rates, throughout the entire sleep cycle and during slow wave sleep, without alteration of ASMs [7].

In this patient, subtle symptoms of late-onset epilepsy, in the form of focal aware seizures (i.e. anxiety and epigastric rising sensation), were present prior to formal OSA diagnosis. However, the development of overt seizures (impaired awareness and bilateral tonic-clonic activity) did not occur until after OSA was diagnosed. It is unclear why the patient's seizures progressed to becoming overtly symptomatic after OSA treatment with CPAP was started. However, his OSA may have become more severe over time, as suggested by several years of worsening symptoms such as snoring and daytime sleepiness, and his seizure threshold may have been lowered by prolonged exposure to the consequences of untreated OSA. In addition, the development of overt seizures occurred in the setting of non-compliance with CPAP therapy, supporting the hypothesis that untreated OSA may facilitate seizures.

The differential diagnosis of the patient's nocturnal episodes includes REM behavior disorder, PTSD related sleep disturbance and non-REM (NREM) parasomnias. As the patient's events lacked vivid dream recall and occurred during the first part of the evening, when NREM rather than REM sleep predominates, REM behavior disorder is less likely. The absence of violent, thrashing movements, and lack of repetitive dreams involving the traumatic event, suggests against PTSD related phenomena [14]. NREM parasomnias, such as confusional arousals, somnambulism (sleep walking) and sleep terrors, occur earlier after sleep onset, similar to this patient's presentation. However, NREM parasomnias tend to be shorter in duration, with less stereotyped behavior and a greater propensity to stand or walk during the event [15].
In this patient, the clinical diagnosis of focal epilepsy was supported by the presence of intermittent sensory symptoms congruent with temporal lobe origin, stereotyped behavior, and timing of nocturnal events, as sleep related seizures are more likely to emerge during the first portion of the evening, corresponding to early stages of sleep (NREM stages 1 and 2) [16]. In addition, temporal lobe seizures have a tendency to generalize during NREM sleep [17].

Further diagnostic testing led to confirmation of epilepsy, including the recording of interictal activity in the right frontotemporal region and subsequent capture of the patient's typical event on EEG. The diagnosis of epilepsy was also reinforced by response of events after initiation of levetiracetam, including improvement of focal aware seizure frequency, and absence of further nocturnal episodes of impaired awareness despite rare CPAP noncompliance.

Several explanations for the causal relationship between OSA and seizures have been proposed. This includes cerebral hypoxemia secondary to sleep disordered breathing, decreased cardiac output, cardiac arrhythmias related to apneic events, and sleep fragmentation with increased arousals during NREM sleep, the latter of which has been associated with activation of epileptiform activity [4,18-20]. However, the exact mechanism(s) responsible for seizure exacerbation in untreated OSA remains unclear and is likely multifactorial.

\section{Conclusions}

Obstructive sleep apnea and epilepsy are prevalent in older adults, and untreated OSA may facilitate seizure susceptibility in these individuals. In people with late-onset epilepsy, modifiable risk factors, such as OSA, should be investigated, as treatment of respiratory events may improve seizure control.

\section{Declarations}

Availability of data and materials: Not applicable

\section{Authors' contributions}

JYC performed critical review of the case, contributed to, and approved, the final manuscript.

\section{Ethics approval and consent to participate}

The study was exempted by the Institutional Review Board

\section{Competing interests}

The author declares that the research was conducted in the absence of any commercial or financial relationships that could be construed as a potential conflict of interest.

\section{Funding}

None

\section{References}

1. Hauser WA, Annegers JF, Kurland LT (1991) Prevalence of epilepsy in Rochester, Minnesota: 1940-1980. Epilepsia 32: 429-445. [Crossref]

2. Dam AM, Fuglsang-Frederiksen A, Svarre-Olsen U, Dam M (1985) Late-onset epilepsy: etiologies, types of seizure, and value of clinical investigation, EEG and computerized tomography scan. Epilepsia 26: 227-231. [Crossref]

3. Perez Lopez JL, Longo J, Quintana F, Diez C, Berciano J (1985) Late onset epileptic seizures: a retrospective study of 250 patients. Acta Neurol Scand 72: 380-384. [Crossref]

4. Devinsky O, Ehrenberg B, Barthlen GM, Abramson HS, Luciano D (1994) Epilepsy and sleep apnea syndrome. Neurology 44: 2060-2064. [Crossref] 
5. Vaughn BV, D’Cruz OF, Beach R, Messenheimer JA (1996) Improvement of epileptic seizure control with treatment of obstructive sleep apnoea. Seizure 5: 73-78. [Crossref]

6. Malow BA, Foldvary-Schaefer N, Vaughn BV, Selwas LM, Chervin RD, et al. (2008) Treating obstructive sleep apnea in adults with epilepsy: a randomized pilot trial. Neurology 71: 572-577. [Crossref]

7. Oliveira AJ, Zamagni M, Dolso P, Bassetti MA, Gigli GL (2000) Respiratory disorders during sleep in patients with epilepsy: effect of ventilatory therapy on EEG interictal epileptiform discharges. Clin Neurophysiol 111: S141-S145. [Crossref]

8. Young T, Palta M, Dempsey J, Skatrud J, Weber S, Badr S (1993) The occurrence of sleep-disordered breathing among middle-aged adults. N Engl J Med 328: 1230-1235. [Crossref]

9. Young T, Shahar E, Nieto FJ, Redline S, Newman AB, et al. (2002) Predictors of sleepdisordered breathing in community-dwelling adults: the Sleep Heart Health Study. Arch Intern Med 162: 893-900. [Crossref]

10. Hollinger P, Khatami R, Gugger M, Hess CW, Bassetti CL (2006) Epilepsy and obstructive sleep apnea. Eur Neurol 55: 74-79. [Crossref]

11. Manni R, Terzaghi M, Arbasino C, Sartori I, Galimberti CA, et al. (2003) Obstructive sleep apnea in a clinical series of adult epilepsy patients: frequency and features of comorbidity. Epilepsia 44: 836-840. [Crossref]

12. Chihorek AM, Abou-Khalil B, Malow B (2007) Obstructive sleep apnea is associated with seizure occurrence in older adults with epilepsy. Neurology 69: 1823-1827. [Crossref]
13. Vendrame M, Auerbach S, Loddenkemper T, Kothare S, Montouris G (2011) Effect of continuous positive airway pressure treatment on seizure control in patients with obstructive sleep apnea and epilepsy. Epilepsia 52: e168-e171. [Crossref]

14. Pillar G, Malhotra A, Lavie P (2000) Post-traumatic stress disorder and sleep-what a nightmare! Sleep Med Rev 4: 183-200. [Crossref]

15. Derry C, Harvey AS, Walker MC, Duncan JS, Berkovic SF (2009) NREM arousal parasomnias and their distinction from nocturnal frontal lobe epilepsy: a video EEG analysis. Sleep 32: 1637-1644. [Crossref]

16. Herman ST, Walczak TS, Bazil CW (2001) Distribution of partial seizures during the sleep-wake cycle: differences by seizure onset site. Neurology 56: 1453-1459. [Crossref]

17. Bazil CW, Walczak TS (1997) Effects of sleep and sleep stage on epileptic and nonepileptic seizures. Epilepsia 38: 56-62. [Crossref]

18. Ferrillo F, Beelke M, Nobili L (2000) Sleep EEG synchronization mechanisms and activation of interictal epileptic spikes. Clin Neurophysiol 111: 65-73. [Crossref]

19. Parrino L, Smerieri A, Spaggiari MC, Terzano MG (2000) Cyclic alternating pattern (CAP) and epilepsy: how a physiological rhythm modulates a pathological event. Clin Neurophysiol 111: S39-S46. [Crossref]

20. Manni R, Zambrelli E, Bellazzi R, Terzaghi M (2005) The relationship between foca seizures and sleep: an analysis of the Cyclic Alternating Pattern. Epilepsy Res 67: 7380. [Crossref]

Copyright: $\odot 2018$ Cheng JY. This is an open-access article distributed under the terms of the Creative Commons Attribution License, which permits unrestricted use, distribution, and reproduction in any medium, provided the original author and source are credited. 